\title{
HLA class II is a factor in cardiovascular morbidity and mortality rates in patients with type 1 diabetes
}

\author{
J. Söderlund • C. Forsblom • J. Ilonen • L. M. Thorn • \\ J. Wadén • M. Parkkonen • P.-H. Groop \\ on behalf of the FinnDiane Study Group
}

Received: 2 March 2012 / Accepted: 22 June 2012 / Published online: 17 August 2012

(C) Springer-Verlag 2012

\begin{abstract}
Aims/hypothesis Patients with type 1 diabetes and kidney disease have a higher risk of cardiovascular events. HLA class II genes are expressed on infiltrated inflammatory cells and smooth-muscle cells in atherosclerotic plaques. We hypothesised that HLA class II haplotypes or genotypes might influence the risk of cardiovascular complications and death in Finnish type 1 diabetic patients.
\end{abstract}

Electronic supplementary material The online version of this article (doi:10.1007/s00125-012-2670-6) contains peer-reviewed but unedited supplementary material, which is available to authorised users.

J. Söderlund $\cdot$ C. Forsblom $\cdot$ L. M. Thorn · J. Wadén •

M. Parkkonen $\cdot$ P.-H. Groop $(\bowtie)$

Folkhälsan Research Center, Biomedicum Helsinki,

University of Helsinki,

Haartmaninkatu 8,

00014 Helsinki, Finland

e-mail: per-henrik.groop@helsinki.fi

J. Söderlund · C. Forsblom • L. M. Thorn · J. Wadén •

M. Parkkonen · P.-H. Groop

Department of Medicine,

Division of Nephrology Helsinki,

Helsinki University Central Hospital,

Helsinki, Finland

J. Ilonen

Immunogenetics Laboratory, University of Turku,

Turku, Finland

J. Ilonen

Department of Clinical Microbiology,

University of Eastern Finland,

Kuopio, Finland

P.-H. Groop

Baker IDI Heart and Diabetes Institute,

Melbourne, Victoria, Australia
Methods We included 3,082 patients with type 1 diabetes from the Finnish Diabetic Nephropathy Study. We analysed the 12 and ten most common HLA II haplo- and genotypes, respectively, using $\chi^{2}$ tests. The positive findings were analysed with three differently adjusted regression models with cardiovascular morbidity and death as endpoints. Different kidney status groups were analysed separately.

Results At baseline, the common (DR1/10)-DQB1*05:01 haplotype (20.4\%) and the (DR1/10)-DQB1*05:01/ $D R B 1 * 04: 01-D Q B 1 * 03: 02$ genotype $(8.7 \%)$ were independently associated with cardiovascular disease in all kidney status groups, except in patients with normal AER. At follow-up (9.45 years; range 0.1-16.1 years), the (DR1/10)$D Q B 1 * 05: 01 / D R B 1 * 04: 01-D Q B 1 * 03: 02$ genotype was associated with cardiovascular mortality rates in patients with normal AER and microalbuminuria.

Conclusions/interpretation The (DR1/10)-DQB1*05:01 haplotype and the (DR1/10)-DQB1*05:01/DRB1*04:01$D Q B 1 * 03: 02$ genotype are independently associated with cardiovascular events and death in Finnish type 1 diabetic patients.

Keywords Cardiovascular disease $\cdot$ Human leucocyte antigen $\cdot$ Mortality $\cdot$ Type 1 diabetes

\begin{tabular}{ll}
\multicolumn{2}{l}{ Abbreviations } \\
AMI & Acute myocardial infarction \\
CVD & Cardiovascular disease \\
eGFR & Estimated GFR \\
ESRD & End-stage renal disease \\
FinnDiane & Finnish Diabetic Nephropathy (Study) \\
hsCRP & High-sensitivity C-reactive protein \\
HSP & Heat shock proteins \\
IQR & Interquartile range \\
oxLDL & Oxidised LDL
\end{tabular}




\section{Introduction}

Death from cardiovascular disease (CVD) is more common in patients with diabetes than in the general population [1]. In patients with type 1 diabetes, the presence of diabetic nephropathy explains most of the risk of premature death [2]. Well-established risk factors for cardiovascular morbidity and death include male sex, smoking, dyslipidaemia, AER, hypertension and overweight, but these factors alone do not fully account for the increased risk of CVD. It is therefore likely that other as yet unidentified factors influence susceptibility to CVD in patients with type 1 diabetes.

The leading cause of CVD is atherosclerosis, which is associated with inflammatory components at all disease stages, including leucocyte infiltration and complement activation [3]. It is unclear whether the HLA class II is also involved in the pathogenesis of atherosclerosis, but HLA is present in atherosclerotic vessel wall plaques, especially on the surface of the accumulated inflammatory cells [4], which when activated can, via IFN- $\gamma$, induce the smooth-muscle cells in the vessel wall plaques to express HLA II genes [5].

The highly polymorphic HLA class II locus on chromosome $6 \mathrm{p} 21.3$ is the strongest genetic factor associated with type 1 diabetes [6]. Some HLA class II alleles confer susceptibility, whereas others protect against the development of type 1 diabetes [7]. Similar properties of the HLA have been observed in connection with the development of coronary artery disease in the general population [8]. Thus genetic variants that influence type 1 diabetes susceptibility might also be associated with the cardiovascular complications seen in patients with type 1 diabetes. We therefore examined whether certain HLA class II haplotypes and genotypes are associated with CVD and cardiovascular mortality rates in Finnish patients with type 1 diabetes.

\section{Methods}

Participants The study included 3,082 well-characterised Finnish adult patients with type 1 diabetes. They were all part of the nationwide, multicentre Finnish Diabetic Nephropathy (FinnDiane) Study, which has been described in detail elsewhere [9]; for details on participating centres, see electronic supplementary materials (ESM) Table 1. In brief, type 1 diabetes was defined as having an age at onset of 40 years or less, with insulin treatment initiated within 1 year of diagnosis. Blood and urine samples were collected for determination of $\mathrm{HbA}_{1 \mathrm{c}}$, lipids, serum creatinine and urinary AER. Standardised questionnaires were completed by the attending physicians to obtain information on clinical events, medication and lifestyle. CVD was defined as acute myocardial infarction (AMI), coronary bypass procedure, peripheral vascular disease or stroke. Diabetic nephropathy was diagnosed when two of three consecutive overnight urine collections showed an AER of $\geq 300 \mathrm{mg}$ per $24 \mathrm{~h}$ or $\geq 200 \mu \mathrm{g} / \mathrm{min}$ (macroalbuminuria). Microalbuminuria was defined as an AER of 30 to $299 \mathrm{mg}$ per $24 \mathrm{~h}$ or 20 to $199 \mu \mathrm{g} / \mathrm{min}$. End-stage renal disease (ESRD) was defined as patients being on dialysis or having received a kidney transplant. The study was approved by the local Ethics Committees of participating study centres. All patients gave informed consent to participate in the study. The study follows the Declaration of Helsinki.

Mortality rate Deaths from any cause through to 17 March 2010 were identified for the entire cohort via a search of the Finnish Cause of Death Registry and evaluated as all-cause and cardiovascular mortality. Death from cardiovascular causes was established when the underlying or immediate cause of death was macrovascular in origin, i.e. given as ICD-10 I21-25 (www.who.int/classifications/icd/en/) for cardiovascular and ICD-10 I60-64 for cerebrovascular deaths.

DNA samples and HLA genotyping Depending on the age of the sample, DNA was isolated from whole blood using a purification kit (Puregene DNA; Gentra Systems, Minneapolis, MN, USA) or the phenol-chloroform method to maximise the yield. HLA genotyping was performed with a sequence-specific hybridisation method using previously described oligonucleotide probes [7]. HLA haplotypes were created on the basis of previously known linkage disequilibrium patterns in whites, with ungenotyped but deduced DR-alleles given in parentheses [7].

Statistical analysis Patients were assigned a binary value (yes/no) for HLA haplotypes and an additional binary value (yes/no) for an HLA haplotype genotype (referred to here as genotype). Due to the highly polymorphic nature of the HLA locus, we analysed only HLA haplotypes with frequencies $\geq 1 \%(n=12)$ and HLA genotypes with frequencies $\geq 2 \%(n=10)$ using separate $\chi^{2}$ tests. With these selection criteria, we captured $92 \%$ of the included patients. For each of the haplotypes or genotypes that showed an association with CVD, associations were explored separately using regression models. For baseline analyses we used logistic regression and for time-to-event (CVD death) analyses Cox regression. These multivariate logistic regression and Cox regression analyses were adjusted using three different models. In Model 1, used for logistic and Cox regression analyses, results were adjusted for the conventional risk factors: sex, duration of diabetes, $\mathrm{BMI}$, systolic $\mathrm{BP}, \mathrm{HbA}_{1 \mathrm{c}}$, $\log _{e}$ triacylglycerol, current smoking and serum creatinine. Model 2 and Model 3 were obtained by using the multivariate backward conditional option, with CVD or CVD death as endpoints, respectively. The $p$ value for inclusion of the 
clinical variables in the models was 0.05 and that for removal was 0.10 . In the process of obtaining the final Model 2 and Model 3 results, the following variables were used: sex, age, age at type 1 diabetes onset, duration of diabetes, $\mathrm{HbA}_{1 \mathrm{c}}$, AER, estimated GFR (eGFR), high-sensitivity Creactive protein (hSCRP), systolic and diastolic blood pressure, pulse pressure, mean arterial pressure, WHR, BMI, triacylglycerol, and total, HDL-, non-HDL- and LDLcholesterol. Model 2 for logistic regression was finally adjusted for: sex, age at type 1 diabetes onset, duration, WHR, systolic BP, eGFR, non-HDL-cholesterol and HDLcholesterol. Model 3 Cox regression was adjusted for: sex, age, pulse pressure, $\log _{e}$ hsCRP, AER, eGFR and HDL. ESRD patients were excluded when fitting Model 2 and Model 3. Different kidney status groups were analysed separately unless otherwise stated, and hence AER and eGFR were excluded from the models. Due to the low number of CVD events and deaths in the normoalbuminuric patient group, we also combined patients with normal AER with microalbuminuric patients to increase statistical power. HLA and hsCRP associations were analysed by a MannWhitney test. SPSS 15 (SPSS, Chicago, IL, USA) was used for the analyses. A value of $p<0.05$ was considered statistically significant. We had sufficient power ( $>98 \%)$ to detect an association for CVD and CVD death in this study population, calculated with http://pngu.mgh.harvard.edu/ purcell/gpc/ [10] (last accessed 3 June 2012).

\section{Results}

At baseline, 315 (10.2\%) of the 3,082 patients had CVD. At follow-up 364 (11.8\%) patients had died, 191 (52.5\% of deaths) from CVD (Table 1). Of the patients who died of cardiovascular causes, 99 (51.8\%) had pre-existing CVD.

Baseline We identified altogether 23 HLA haplotypes and 115 HLA genotypes (data not shown), but included only the 12 and ten most frequent haplo- and genotypes for further analyses, respectively (Table 2). At baseline, the (DR1/10)$D Q B 1 * 05: 01$ haplotype was enriched in patients with CVD (26.6\% vs $19.6 \% ; p=0.021$ ). Using Model 1 (ESRD patients excluded), (DR1/10)-DQB1*05:01 was associated with CVD (OR 1.65, 95\% CI 1.13, 2.40; $p=0.009$ ). Separate analysis of

Table 1 Patient characteristics

Unless otherwise stated, values are presented as means (SD), except those for serum creatinine, urinary AER, triacylglycerol and hsCRP, which are given as median (IQR)

${ }^{*} p<0.05,{ }^{* * *} p<0.001$ compared with undeceased patients without CVD

\begin{tabular}{|c|c|c|c|}
\hline Characteristic & Alive, no CVD & CVD & CVD death \\
\hline$n$ & 2446 & 315 & 191 \\
\hline Male $(\%)$ & 49.5 & 52.0 & $63.9^{* * *}$ \\
\hline Age (years) & $36.4(11.1)$ & $49.8(9.2)^{* * *}$ & $48.2(9.79)^{* * *}$ \\
\hline Age at onset (years) & $14.4(8.6)$ & $14.33(8.8)$ & $14.14(9.23)$ \\
\hline Duration of diabetes (years) & $22.0(10.9)$ & $35.4(8.8)^{* * *}$ & $34.08(8.71)^{* * *}$ \\
\hline Diabetic nephropathy (\%) & 17.4 & 26.0 & $76.4^{* * *}$ \\
\hline Current smoking (\%) & 23.0 & 23.1 & 24.2 \\
\hline $\operatorname{BMI}\left(\mathrm{kg} / \mathrm{m}^{2}\right)$ & $25.1(3.4)$ & $25.4(3.9)$ & $24.76(3.74)$ \\
\hline WHR, men & $0.91(0.07)$ & $0.95(0.07)^{* * *}$ & $0.96(0.07)^{* * *}$ \\
\hline WHR, women & $0.81(0.06)$ & $0.87(0.08)^{* * *}$ & $0.85(0.08)^{* * *}$ \\
\hline $\mathrm{HbA}_{1 \mathrm{c}}(\%)$ & $8.41(1.41)$ & $8.71(1.45)^{* * *}$ & $8.87(1.67)^{* * *}$ \\
\hline $\mathrm{HbA}_{1 \mathrm{c}}(\mathrm{mmol} / \mathrm{mol})$ & 68.42 & 71.70 & 73.45 \\
\hline Systolic BP (mmHg) & $132(17)$ & $148(21)^{* * *}$ & $150(22)^{* * *}$ \\
\hline Diastolic BP (mmHg) & $80(10)$ & $81(11)^{*}$ & $83(12)^{* * *}$ \\
\hline Pulse pressure $(\mathrm{mmHg})$ & $53(15)$ & $67(19)^{* * *}$ & $68(19)^{* * *}$ \\
\hline $\begin{array}{l}\text { Mean arterial pressure } \\
(\mathrm{mmHg})\end{array}$ & $97(11)$ & $104(12)^{* * *}$ & $105(13)^{* * *}$ \\
\hline Serum creatinine $(\mu \mathrm{mol} / \mathrm{l})$ & $84(72-97)$ & $120(94-209)^{* * *}$ & $142(97-285)^{* * *}$ \\
\hline eGFR $\left(\mathrm{ml} \mathrm{min} \min ^{-1} 1.73 \mathrm{~m}^{-2}\right)$ & $97.1(33.1)$ & $57.8(29.8)^{* * *}$ & $57.2(29.5)^{* * *}$ \\
\hline Urinary AER (mg/24 h) & $11.0(6.0-43.0)$ & $42.0(10.0-298.5)^{* * *}$ & $96.5(20.8-667.0)^{* * *}$ \\
\hline Triacylglycerol $(\mathrm{mmol} / \mathrm{l})$ & $1.01(0.76-1.43)$ & $1.31(0.99-1.88)^{* * * *}$ & $1.42(1.06-2.11)^{* * *}$ \\
\hline Total cholesterol $(\mathrm{mmol} / \mathrm{l})$ & $4.93(0.95)$ & $5.09(0.94)$ & $5.43(1.27)^{* * *}$ \\
\hline HDL-cholesterol (mmol/1) & $1.32(0.37)$ & $1.18(0.39)^{* * *}$ & $1.15(0.399)^{* * * *}$ \\
\hline LDL-cholesterol (mmol/1) & $3.07(0.84)$ & $3.19(0.84)$ & $3.46(0.974)^{* * * *}$ \\
\hline $\begin{array}{l}\text { Non-HDL-cholesterol } \\
(\mathrm{mmol} / \mathrm{l})\end{array}$ & $3.63(0.99)$ & $3.89(0.99)^{*}$ & $4.28(1.29)^{* * *}$ \\
\hline hsCRP (nmol/1) & $18.57(11.24-36.86)$ & $27.24(15.14-59.43)^{* * *}$ & $24.10(14.76-62.10)^{* * *}$ \\
\hline
\end{tabular}


Table 2 Frequencies of the most common HLA haplotypes and genotypes in the FinnDiane Study

\begin{tabular}{|c|c|c|c|c|c|}
\hline Haplotype $^{\mathrm{a}}$ & $n$ & $\%$ & HLA genotype ${ }^{b}$ & $n$ & $\%$ \\
\hline$D R B 1 * 04: 01-D Q B 1 * 03: 02$ & 1791 & 58.1 & $(D R 3)-D Q A 1 * 05-D Q B 1 * 02 / D R B 1 * 04: 01-D Q B 1 * 03: 02$ & 578 & 18.8 \\
\hline$(D R 3)-D Q A 1 * 05-D Q B 1 * 02$ & 1255 & 40.7 & $(D R 1 / 10)-D Q B 1 * 05: 01 / D R B 1 * 04: 01-D Q B 1 * 03: 02$ & 269 & 8.7 \\
\hline$(D R 1 / 10)-D Q B 1 * 05: 01$ & 629 & 20.4 & $(D R 8)-D Q B 1 * 04 / D R B 1 * 04: 01-D Q B 1 * 03: 02$ & 249 & 8.1 \\
\hline$D R B 1 * 04: 04-D Q B 1 * 03: 02$ & 509 & 16.5 & $D R B 1 * 04: 01-D Q B 1 * 03: 02 / D R B 1 * 04: 01-D Q B 1 * 03: 02$ & 199 & 6.5 \\
\hline$(D R 8)-D Q B 1^{*} 04$ & 478 & 15.5 & $D R 3-D Q A 1 * 05-D Q B 1 * 02 / D R B 1 * 04: 04-D Q B 1 * 03: 02$ & 192 & 6.2 \\
\hline$(D R 13)-D Q B 1 * 06: 04$ & 269 & 8.7 & $(D R 1 / 10)-D Q B 1 * 05: 01 /(D R 3)-D Q A 1 * 05-D Q B 1 * 02$ & 129 & 4.2 \\
\hline$(D R 9)-D Q A 1 * 03-D Q B 1 * 03: 03$ & 177 & 5.7 & $(D R 13)-D Q B 1 * 06: 04 / D R B 1 * 04: 01-D Q B 1 * 03: 02$ & 110 & 3.6 \\
\hline$(D R 7)-D Q A 1 * 02: 01-D Q B 1 * 02$ & 147 & 4.8 & $(D R 3)-D Q A 1 * 05-D Q B 1 * 02 /(D R 3)-D Q A 1 * 05-D Q B 1 * 02$ & 107 & 3.5 \\
\hline$(D R 4)-D Q A 1 * 03-D Q B 1 * 03: 01$ & 135 & 4.4 & $D R B 1 * 04: 01-D Q B 1 * 03: 02 / D R B 1 * 04: 04-D Q B 1 * 03: 02$ & 89 & 2.9 \\
\hline$(D R 13)-D Q B 1 * 06: 03$ & 126 & 4.1 & $(D R 13)-D Q B 1 * 06: 03 / D R B 1 * 04: 01-D Q B 1 * 03: 02$ & 82 & 2.7 \\
\hline$(D R 11 / 12 / 13)-D Q A 1 * 05-D Q B 1 * 03: 01$ & 123 & 4.0 & & & \\
\hline$(D R 15)-D Q B 1 * 06: 02$ & 39 & 1.3 & & & \\
\hline
\end{tabular}

${ }^{\mathrm{a}}$ Frequency $\geq 1 \%$

${ }^{\mathrm{b}}$ Frequency $\geq 2 \%$

the kidney status groups revealed that the (DR1/10)$D Q B 1^{*} 05: 01$ haplotype was independently associated with CVD in macroalbuminuric patients adjusted as for Model 1 (OR 2.11, $p=0.012$ ) and Model 2 (OR 2.09, $p=0.019$ ). The (DR1/10)-DQB1*05:01/DRB1*04:01-DQB1*03:02 genotype was associated with CVD in patients with microalbuminuria at baseline when adjusted as for Model 1 (OR 4.05, $p=0.02$ ) and Model 2 (OR 4.86, $p=0.023$ ), and also when normo- and microalbuminuric patients were combined (Table 3). In the ESRD group, the (DR1/10)-DQB1*05:01/ $D R B 1 * 04: 01-D Q B 1 * 03: 02$ genotype was enriched in patients without CVD events $(10.9 \%$ vs $5.1 \% ; p=0.093)$ and was associated with decreased risk of CVD after adjustment as for Model 2 (OR 0.171, $p=0.034$ ) (Table 3). We did not observe any significant associations for protective HLA haplo- or genotypes. We also analysed the identified risk HLA haplo- and genotypes by hsCRP levels separately for each kidney status group, observing higher hsCRP levels in the normoalbuminuric patient group for carriers of (DR1/10)$D Q B 1 * 05: 01$ (median 19.05, interquartile range [IQR] $11.62-36.00$ vs 16.76 , IQR $10.00-32.10 ; p=0.023)$ and (DR1/10)-DQB1*05:01/DRB1*04:01-DQB1*03:02 (20.67, IQR 12.67-40.19 vs17.05, IQR 10.10-32.38; $p=$ $0.015)$.

Follow-up The mean follow-up time was 9.45 years (range $0.1-16.1$ years). The $(D R 1 / 10)-D Q B 1 * 05: 01 / D R B 1 * 04: 01$ $D Q B 1^{*} 03: 02$ genotype was associated with cardiovascular death in the patient group combining normo- and microalbuminuric patients when adjusted with Model 1 (HR 3.92, logrank $p=0.00021$ ) and Model 3 (HR 3.20, logrank $p=$ 0.002) (Table 3). Of the 45 patients who died from CVD in the normo- and microalbuminuric patient group, ten $(22.2 \%)$ had (DR1/10)-DQB1*05:01/DRB1*04:01-DQB1*03:02, making this the most prevalent genotype. By comparison, the (DR3)-DQA1*05-DQB1*02/DRB1*04:01-DQB1*03:02 genotype was the second most common with a prevalence of $17.8 \%$ in this patient group, whereas normally it is the most common genotype among our patients (18.8\%). We also inserted pre-existing CVD as a covariate in the Cox models. In Model 1 and Model 3, the (DR1/10)-DQB1*05:01/ $D R B 1 * 04: 01-D Q B 1 * 03: 02$ genotype was significantly associated with CVD death in the patient group combining normoand microalbuminuric patients (Model 1: HR 2.89, 95\% CI 1.40, 5.98; logrank $p=0.004$; Model 3: HR 2.31, 95\% CI 1.08, 4.94; logrank $p=0.032$ ). We did not observe any significant associations for protective HLA haplo- or genotypes.

\section{Discussion}

At baseline and prospectively, the presence of the common (DR1/10)-DQB1*05:01 haplotype (20.7\%), alone or in combination with $D R B 1 * 04: 01-D Q B 1 * 03: 02$, is associated with cardiovascular events, as well as with cardiovascular mortality rates in type 1 diabetes. We applied different multivariate regression models, in addition to more conventional risk factor analysis, and in both cases the (DR1/10)$D Q B 1 * 05: 01$ haplotype or the (DR1/10)-DQB1*05:01/ $D R B 1^{*} 04: 01-D Q B 1 * 03: 02$ genotype was a significant independent risk factor for CVD or cardiovascular death. To our knowledge, the FinnDiane Study has to date the largest collection of adult type 1 diabetes patients, making it ideal for our study setting of CVD and CVD death manifesting later in life. Unfortunately, the difficulty of finding a sufficiently large replication cohort has prevented us from replicating our findings. Nevertheless, other studies, although not performed in participants with diabetes, provide supporting evidence of the 


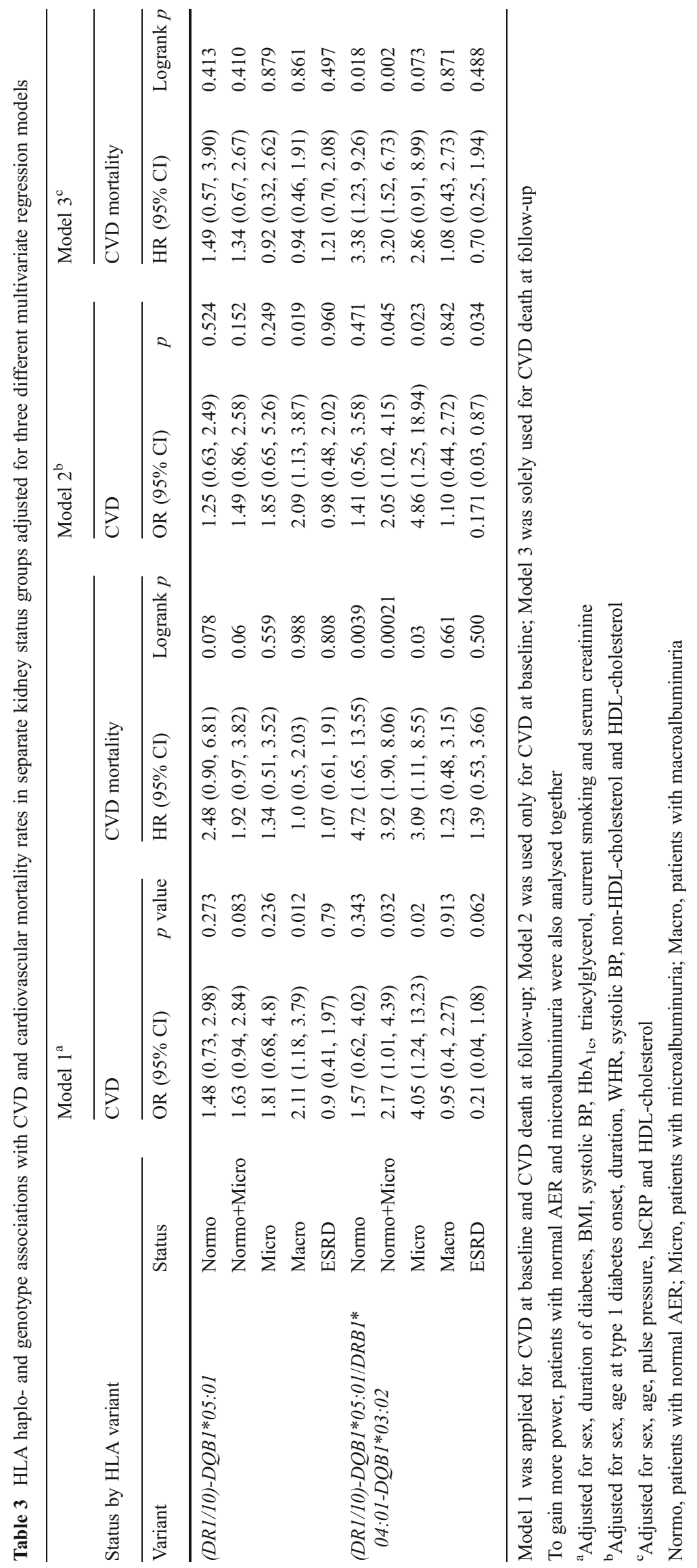


involvement of the haplotype and genotype identified by us in CVD and CVD death. Thus the (DR1/10)-DQB1*05:01 haplotype was associated with coronary artery disease in the general Finnish population [8] and the $H L A-D R B 1^{*} 01: 01$ $D Q A 1^{*} 01-D Q B 1 * 05$ haplotype was associated with AMI in a non-diabetic Swedish cohort [11]. A Chinese study also linked the $D R B 1^{*} 01$ gene to coronary heart disease [12]. A study by Farragher et al [13] used a research setting analogous to ours to study mortality rates and CVD in patients with two different autoimmune diseases: rheumatoid arthritis and inflammatory polyarthritis. A common denominator between type 1 diabetes, rheumatoid arthritis and inflammatory polyarthritis seems to be that HLA is a causal risk factor for the development of all three disorders. Interestingly, the genotype we identified as being associated with cardiovascular death, namely (DR1/10)-DQB1*05:01/DRB 1*04:01-DQB1*03:02, was the same as that shown by Farragher et al to influence CVD mortality rates in their patients [13]. Some other studies have also shown similar results for $(D R 1 / 10)-D Q B 1 * 05: 01$ and/or $D R B 1^{*} 04: 01-D Q B 1^{*} 03: 02$ with regard to CVD and death in connection with rheumatoid arthritis $[14,15]$. A recent study performed in a Finnish population showed that the haplotype containing $H L A-D R B 1^{*} 01$ is associated with ST-elevation myocardial infarction [16]. Taken together, these data suggest that $H L A-(D R 1 / 10)-D Q B 1 * 05: 01$, alone or in combination with $D R B 1^{*} 04: 01-D Q B 1^{*} 03: 02$, confers a higher risk of cardiovascular events and death in the general population, in patients with autoimmune diseases and specifically in those with type 1 diabetes.

Findings for the haplo- or genotypes, and CVD and CVD mortality rates, differed on the basis of diabetic kidney disease status, with associations not consistently predisposing to CVD in ESRD patients. The inverse relationship between CVD events and the (DR1/10)-DQB1*05:01/ $D R B 1 * 04: 01-D Q B 1 * 03: 02$ genotype in ESRD patients supports our findings in the other kidney status groups, and could be due to survival bias. It can be speculated that patients who develop ESRD might have survived cardiovascular events and avoided death at the macroalbuminuric stage, and may therefore have a different genetic make-up compared with the other kidney status groups. In this case, the risk HLA genotype for CVD may be protective in ESRD, because patients with the (DR1/10)-DQB1*05:01/ $D R B 1 * 04: 01-D Q B 1 * 03: 02$ genotype would normally have died at an earlier stage of their kidney disease [17].

HLA class II expression is upregulated or altered in many autoimmune diseases and this could partly explain the higher prevalence of CVD in autoimmunity observed in a previous review [18]. A growing body of evidence links several factors of the immune system to atherosclerosis; indeed, atherosclerosis itself has even been proposed to be an inflammatory autoimmune disease [19]. In patients with CVD, autoantigens are frequently found, especially heat shock proteins (HSPs) and oxidised LDL (oxLDL) [20, 21]. Internalised oxLDL is presented by HLA II molecules on the infiltrated macrophages to $\mathrm{CD}^{+} \mathrm{T}$ cells, which further enhance the ongoing inflammation process in the vessels by secreting inflammatory cyto- and chemokines $[19,22]$. IFN- $\gamma$ is secreted by monocytes/macrophages and also by $\mathrm{CD}^{+} \mathrm{T}$ cells [19], and is capable of inducing expression of the mostly absent $H L A-D Q$ and $H L A-D P$ genes in vessels; $H L A-D R$ is nevertheless constitutively expressed on endothelial cells [18]. It could be hypothesised that the $(D R 1 / 10)-D Q B 1 * 05: 01 / D R B 1 * 04: 01-D Q B 1 * 03: 02$ genotype, which was found to be associated with CVD and CVD death, might be upregulated in the vessel walls or carry (HLA) genes that have a specific affinity for oxLDL or HSP60, or as yet unidentified autoimmune epitopes, thus exacerbating the pro-atherosclerotic inflammatory process. The higher CRP levels seen in our patients with normoalbuminuria and the risk haplo- and genotypes are suggestive of an exacerbated inflammation process. This inflammatory activation could possibly contribute to the development of CVD and CVD death in patients with these risk HLA haplo- and genotypes.

Although a plausible biological link exists between HLA class II genes and atherosclerosis, it should be recognised that the linkage disequilibrium and haplotype structure of the HLA locus also enable the observed association to be located elsewhere on chromosome 6p. A recent genomewide association study identified a risk locus for coronary artery disease in the HLA locus [23], highlighting the possibility that our finding could also be located in the extended HLA haplotype. HLA class I, as well as complement and other genes of the immune system are possible candidates for the associations [8] and this requires further study.

We did not identify any protective haplotypes, not even the (DR7)-DQA1*02:01-DQB1*02 haplotype identified by Palikhe et al in the Finnish population [8] for CVD or CVD death, or haplotypes identified in a Chinese population [24]. Possibly, the protective haplotypes or genotypes were not detectable in our study setting, due to their low frequencies or insufficient statistical power to detect their effect.

The main strength of this study is that it draws on complete follow-up in a well-characterised patient collection. One potential limitation is that the number of individuals affected by CVD is relatively small and this number is further reduced when the different HLA haplotypes and genotypes are analysed. However, although the numbers are small, the presence of the high CVD risk HLA (DR1) 10)-DQB1*05:01 haplotype or (DR1/10)-DQB1*05:01/ $D R B 1 * 04: 01 D Q B 1 * 03: 02$ genotype is nevertheless a significant risk factor, in addition to conventional factors, for carriers to suffer a CVD event or premature cardiovascular death. Therefore, it may be worth monitoring patients with the susceptibility HLA haplotype or genotype even more 
carefully, because they seem to increase the risk of cardiovascular death, even in patients with no kidney disease or minor changes in their kidneys. However, these observational findings first need to be confirmed in future experimental settings.

To summarise, we identified HLA class II haplo- and genotypes that are associated with CVD and CVD death in a large Finnish type 1 diabetes cohort, regardless of the kidney status of the patients.

Acknowledgements We acknowledge the skilled assistance of A. Sandelin, T. Soppela and A.-R. Salonen in Helsinki, and of P. Nurmi in Turku. We also acknowledge all the physicians and nurses at each participating study centre for the collection of patients for the FinnDiane Study (details, see ESM Table 1).

Funding This study was supported by grants from the Folkhälsan Research Foundation, the Wilhelm and Else Stockmann Foundation, the Waldemar von Frenckell Foundation, the Liv och Hälsa Foundation and the European Commission (contract Health-F2-2008-223211 CEED3).

Duality of interest The authors declare that there is no duality of interest associated with this manuscript.

Contribution statement JS researched the data and wrote the manuscript. CF, JI, LMT, JW and PHG contributed to the acquisition of data and the discussion, and reviewed the manuscript. MP contributed to the acquisition of data and reviewed the manuscript. All authors have read the final version of the paper

\section{References}

1. Morrish NJ, Wang SL, Stevens LK, Fuller JH, Keen H (2001) Mortality and causes of death in the WHO Multinational Study of Vascular Disease in Diabetes. Diabetologia 44(Suppl 2):S14-S21

2. Groop PH, Thomas MC, Moran JL et al (2009) The presence and severity of chronic kidney disease predicts all-cause mortality in type 1 diabetes. Diabetes 58:1651-1658

3. Hansson GK, Libby P, Schonbeck U, Yan ZQ (2002) Innate and adaptive immunity in the pathogenesis of atherosclerosis. Circ Res 91:281-291

4. Hansson GK, Robertson AK, Soderberg-Naucler C (2006) Inflammation and atherosclerosis. Annu Rev Pathol 1:297-329

5. Ross R (1999) Atherosclerosis - an inflammatory disease. N Engl J Med 340:115-126

6. Barrett JC, Clayton DG, Concannon P et al (2009) Genome-wide association study and meta-analysis find that over 40 loci affect risk of type 1 diabetes. Nat Genet 41:703-707

7. Hermann R, Turpeinen H, Laine AP et al (2003) HLA DR-DQencoded genetic determinants of childhood-onset type 1 diabetes in Finland: an analysis of 622 nuclear families. Tissue Antigens 62:162-169

8. Palikhe A, Sinisalo J, Seppanen M, Valtonen V, Nieminen MS, Lokki ML (2007) Human MHC region harbors both susceptibility and protective haplotypes for coronary artery disease. Tissue Antigens 69:47-55

9. Thorn LM, Forsblom C, Fagerudd J et al (2005) Metabolic syndrome in type 1 diabetes: association with diabetic nephropathy and glycemic control (the FinnDiane study). Diabetes Care 28:2019-2024

10. Purcell S, Cherny SS, Sham PC (2003) Genetic power calculator: design of linkage and association genetic mapping studies of complex traits. Bioinformatics 19:149-150

11. Bjorkbacka H, Lavant EH, Fredrikson GN et al (2010) Weak associations between human leucocyte antigen genotype and acute myocardial infarction. J Intern Med 268:50-58

12. Sun W, Cui Y, Zhen L, Huang L (2011) Association between HLA-DRB1, HLA-DRQB1 alleles, and CD4(+)CD28(null) T cells in a Chinese population with coronary heart disease. Mol Biol Rep 38:1675-1679

13. Farragher TM, Goodson NJ, Naseem H et al (2008) Association of the HLA-DRB1 gene with premature death, particularly from cardiovascular disease, in patients with rheumatoid arthritis and inflammatory polyarthritis. Arthritis Rheum 58:359-369

14. Gonzalez-Gay MA, Gonzalez-Juanatey C, Lopez-Diaz MJ et al (2007) HLA-DRB1 and persistent chronic inflammation contribute to cardiovascular events and cardiovascular mortality in patients with rheumatoid arthritis. Arthritis Rheum 57:125-132

15. Mattey DL, Thomson W, Ollier WE et al (2007) Association of DRB1 shared epitope genotypes with early mortality in rheumatoid arthritis: results of eighteen years of follow up from the early rheumatoid arthritis study. Arthritis Rheum 56:1408-1416

16. Paakkanen R, Lokki ML, Seppanen M, Tierala I, Nieminen MS, Sinisalo J (2012) Proinflammatory HLA-DRB $1 * 01$-haplotype predisposes to ST-elevation myocardial infarction. Atherosclerosis 221:461-466

17. Boger CA, Stubanus M, Haak T et al (2007) Effect of MTHFR C677T genotype on survival in type 2 diabetes patients with endstage diabetic nephropathy. Nephrol Dial Transplant 22:154-162

18. Turesson C (2004) Endothelial expression of MHC class II molecules in autoimmune disease. Curr Pharm Des 10:129-143

19. Sitia S, Tomasoni L, Atzeni F et al (2010) From endothelial dysfunction to atherosclerosis. Autoimmun Rev 9:830-834

20. Yla-Herttuala S, Palinski W, Butler SW, Picard S, Steinberg D, Witztum JL (1994) Rabbit and human atherosclerotic lesions contain $\operatorname{IgG}$ that recognizes epitopes of oxidized LDL. Arterioscler Thromb 14:32-40

21. Burut DF, Karim Y, Ferns GA (2010) The role of immune complexes in atherogenesis. Angiology 61:679-689

22. Hansson GK, Hermansson A (2011) The immune system in atherosclerosis. Nat Immunol 12:204-212

23. Davies RW, Wells GA, Stewart AF et al (2012) A genome wide association study for coronary artery disease identifies a novel susceptibility locus in the major histocompatibility complex. Circ Cardiovasc Genet 5:217-225

24. Liu B, Xiong L, Tian C et al (2012) HLA-DRB1*12:02:01 plays a protective role against coronary artery disease in women of southern Han Chinese descent. Hum Immunol 73:122-126 